\title{
ON FUNCTION SPACES OF STRATIFIABLE SPACES AND COMPACT SPACES
}

\author{
CARLOS J. R. BORGES ${ }^{1}$
}

1. Introduction. Throughout, for any topological spaces $X$ and $Y$, $Y^{X}$ will denote the space of continuous functions from $X$ to $Y$ with the compact-open topology,$^{2}$ unless otherwise stated. Since stratifiable $^{3}$ spaces, which we studied in [2], have many of the desirable properties of metrizable spaces (every $\mathrm{CW}$-complex of Whitehead is stratifiable-see Theorem 7.2 in [2] or Theorem 8.1 in [3]; furthermore, it is easily seen that metrizable spaces are stratifiable and every stratifiable space is paracompact and perfectly normal), we naturally questioned whether $Y^{X}$ is stratifiable given that $X$ is compact Hausdorff and $Y$ is stratifiable. We will now answer this question negatively. However, the stratifiable space $Y$ of our example is not a CW-complex and thus the following question still remains unanswered: Is $K^{X}$ stratifiable whenever $X$ is compact Hausdorff and $K$ is a CW-complex? Whenever $K$ is a countable CW-complex and $X$ is compact metrizable we will however show that $K^{\boldsymbol{X}}$ is a cosmic ${ }^{4}$ space (hence $K^{\boldsymbol{x}}$ is hereditarily Lindelöf, thus paracompact, and hereditarily separable) whenever $K^{x}$ has the pointwise topology or the compact-open topology.

We will also give a negative answer to the following question of Stone [9]: Is $Y^{I}$ a normal space whenever $Y$ is compact Hausdorff and finite-dimensional (in the covering sense)?

Throughout we use the terminology of Kelley [6], except that all our topological spaces are $T_{1}$.

2. Theorems and proofs. Throughout this section, let $I$ denote the closed unit interval.

Theorem 1. There exists a stratifiable space $X$ such that $X^{I}$ is not a normal space. Furthermore $X$ is a 2-dimensional (in any sense-ind, Ind, dim) cosmic space. ${ }^{4}$

Received by the editors January 8, 1965 and, in revised form, October 28, 1965.

1 This research was partially supported by the NSF Grant GP-4770.

${ }^{2}$ For $K$ a compact subset of $X$ and $V$ an open subset of $Y, W(K, V)=\left\{f \in Y^{x}\right\}$ $f(K) \subset V\}$. A subbase for the compact-open topology in $Y^{x}$ is the family of all $W(K, V)$, with $K$ compact and $V$ open $(K \subset X, V \subset Y)$.

${ }^{3}$ A topological space $X$ is a stratifiable space if $X$ is $T_{1}$ and, to each open $U \subset X$, one can assign a sequence $\left\{U_{n}\right\}_{n=1}^{\infty}$ of open subsets of $X$ such that, for all $n, U_{n}^{-} \subset U$, $U_{n} \subset V_{n}$ whenever $U \subset V$, and $\cup_{n=1}^{\infty} U_{n}=U$. This definition is equivalent to Definition 1.3 in [3] (thus our stratifiable spaces are the same as the $M^{3}$-spaces of Ceder [3]).

4 A topological space $X$ is cosmic if it is the continuous image of a separable metrizable space. 
Proof. Let $X=\{(x, y) \mid x$ and $y$ are real numbers and $y \geqq 0\}$. For each $(x, 0) \in X$ and angle $\alpha, 0<\alpha<\pi$, let $L(x, \alpha)$ be the ray through $(x, 0)$ lying in $X$ whose angle of inclination with the positive $x$-axis is $\alpha$. For each $(x, y) \in X$, with $y \neq 0$, and real number $r>0$, let $D((x, y), r)$ denote the circular disc centered at $(x, y)$ and with radius $r$, and let $C((x, y), r)$ denote the boundary of $D((x, y), r)$.

Let $X$ have the topology $\tau$ with the following neighborhood system:

(a) If $(x, y) \in X$ and $y \neq 0$, a neighborhood of $(x, y)$ is any $D((x, y), r)$ with $0<r<y$.

(b) A neighborhood of $(w, 0) \in X$ is a set of the form $N(w, \sigma, \partial)$ $=S(w, \pi-\sigma, \partial) \cup\{(w, 0)\} \cup S(w, \sigma, \partial)$, where

$$
S(w, \alpha, \partial)=\{(x, y) \in X|| x-w \mid<\partial, y<(x-w) \tan \alpha\}
$$

(where $\partial>0,0<\alpha<\pi$, and $\alpha \neq \pi / 2)$. Note that $S(w, \alpha, \partial)$ is a region bounded by a right triangle, lying to the right of $(w, 0)$ if $0<\alpha<\pi / 2$, to the left of $(w, 0)$ if $\pi / 2<\alpha<\pi$.

It is easily seen that $(X, \tau)$ is stratifiable: ${ }^{5}$ For each open $U \subset X$ and positive integer $n$, let $U_{n}=U_{n}^{\prime} \cup U_{n}^{\prime \prime}$, where

$$
\begin{aligned}
U_{n}^{\prime} & =U\{D((x, y), 1 / 2 n) \mid D((x, y), 1 / n) \subset U\}, \\
U_{n}^{\prime \prime} & =U\{N(x, 1 / 2 n, 1 / 2 n) \mid N(x, 1 / n, 1 / n) \subset U\} .
\end{aligned}
$$

A simple argument shows that $\left(U_{n}^{\prime \prime}\right)-\subset U$. Obviously, $\left(U_{n}^{\prime}\right)-\subset U$, $\cup_{n=1}^{\infty} U_{n}=U$ and $U_{n} \subset V_{n}$ whenever $U \subset V$. Consequently $(X, \tau)$ is stratifiable (see footnote 1 ).

It is clear that ind $X=2$, and it can be seen that $\operatorname{dim} X=2=\operatorname{Ind} X$. Letting $Y=\{(x, y) \in X \mid y>0\}$ and $R=X-Y$ then both $Y$ and $R$ are separable metrizable subspaces of $X$ and thus $X$ is the one-to-one continuous image of the topological sum ${ }^{6}$ of $Y$ and $R$ (See Example 12.1 in [8]). Hence $X$ is a cosmic space.

We will now show that $X^{I}$ is not a normal space: Let $F$ be the set of all functions $f_{x} \in X^{I}(x \in X)$ such that $f_{x}$ maps $I$ in a "natural fashion" onto the arc $C_{x}$ of the circle $C((x, 1), 1)$ with unit length, and such that $f_{x}\left(\frac{1}{2}\right)=(x, 0)$ (simply lay the unit interval $I$ around $C((x, 1), 1)$ so that the center of $I$ coincides with the point $(x, 0))$. Then

(a) $X^{I}$ is not hereditarily separable (this was first observed by Professor E. A. Michael) : $F$ is a discrete subspace of $X^{I}$ since

${ }^{5}$ Actually one can show that $X$ is $M_{1}$ (see Definition 1.1 in [3]) by the method of proof used in Example 9.2 of [3].

6 A topological space $M$ is the topological sum of the family $\left\{X_{\alpha}\right\}_{\alpha \in L}$ of topological spaces if $M=\cup_{\alpha \in L} X_{\alpha} \times\{\alpha\}$ with $X_{\alpha}\{\alpha\}$ homeomorphic to $X_{\alpha}$ for each $\alpha \in L$. 


$$
\left\{f_{x}\right\}=W(I, N(x, \pi / 6,1)) \cap W(\{0\}, S(x, 5 \pi / 6,1)) \cap W(\{1\}, S(x, \pi / 6,1))
$$

for each $x \in X$. Since $F$ is discrete and uncountable, $X^{I}$ is not hereditarily separable.

(b) $F$ is a closed subset of $X^{I}:$ By $2.4,2.5$ and 4.71 in [1], a net $\left\{f_{x}\right\}_{x \in A}$ converges to $h \in X^{I}$ if and only if $\left\{f_{x}\right\}_{x \in A}$ converges continuously to $h$. Hence, if $\left\{f_{x}\right\}_{x \in A}$ converges continuously to $h \in X^{I}$ then $\left\{f_{x}(t)\right\}_{x \in A}$ converges to $h(t)$ for each $t \in I$, and thus $h \in F$. (Let $a=h\left(\frac{1}{2}\right)$. By the definition of the functions $f_{x} \in F$ it is clearly seen that for each $t \in I,\left\{f_{x}(t) \mid x\right.$ is a real number $\}$ is a subset of a horizontal straight line. Thus one can easily see that $h=f_{a} \in F$ : Certainly, for each $t \in I, h(t)$ and $f_{a}(t)$ are in the same horizontal line since $h(t)$ is the limit point of the net $\left\{f_{x}(t)\right\}_{x \in A}$; thus it is obvious that $h(t)=f_{a}(t)$.)

(c) There exists a separable subspace $Z$ of $X^{I}$ which contains $F$ : For each pair $(r, t)$ of rational numbers with $r<t$ and $t-r<1$, let $f_{r t}$ be the function in $X^{I}$ such that $f_{r t}$ maps the closed interval $I_{r}$ $=[0,(1+r-t) / 2]$ in a "natural fashion" on to the $\operatorname{arc} C_{r}$ of $C((r, 1), 1)$ with length $(1+r-t) / 2$ and $f_{r t}((1+r-t) / 2)=(r, 0), f_{r t}$ similarly maps $I_{t}=[(1+t-r) / 2,1]$ onto the arc $C_{t}$ of $C((t, 1), 1)$ with length $(1+r-t) / 2$ and $f_{r t}((1+t-r) / 2)=(t, 0)$, and $f_{r t}$ maps $I-\left(I_{r} \cup U_{t}\right)$ onto $\{(x, 0) \mid r<x<t\}$ in a "natural fashion."

Now let $Z=F \cup D$, where $D=\left\{f_{r i} \in X^{I} \mid r\right.$ and $t$ are rational numbers, $r<t$ and $t-r<1\}$. We show that $D$ is a dense subset of $Z$ : Given any function $f_{x} \in F$, let $\{r(n)\}_{n=1}^{\infty}$ be a decreasing sequence of rational numbers converging to $x$ and let $\{q(n)\}_{n=1}^{\infty}$ be an increasing sequence of rational numbers converging to $x$, such that $r(1)-q(1)<1$. Then the sequence $\left\{f_{q(n) r(n)}\right\}_{n=1}^{\infty}$ of functions in $D$ continuously converges to $f_{x}$ : Let $\left\{t_{\nu}\right\}_{\nu \in C}$ be a net of points in $I$ which converges to $t \in I$. If $t \neq \frac{1}{2}$ then $f_{x}(t) \notin\{(x, 0) \mid x$ is a real number $\}$ and obviously the net $\left\{g_{n}\left(t_{v}\right)\right\}$ converges to $f_{x}$, where $g_{n}=f_{q(n) r(n)}$ for each $n$, since the subspace $\{(x, y) \in X \mid y>0\}$ of $X$ is also a subspace of the cartesian plane with the usual topology. If $t=\frac{1}{2}$, then $f_{x}(t)=(x, 0)$ and one easily sees that $\left\{g_{n}\left(t_{v}\right)\right\}$ converges to $f_{x}(t)$ from the definition of the functions $g_{n}$ and the neighborhood system of $(x, 0)$. Since $D$ is countable then $Z$ is separable.

(d) $X^{I}$ is not a normal space: Let $E$ be the closure of $Z$ in $X^{I}$. Then $E$ is separable and contains a subset $F$ of cardinality $2^{N_{0}}$ without a limit point in $E$ (since $F$ is a discrete closed subspace of $X^{I}$ ). By Theorem 1 in [5], $E$ is not normal.

7 The net $\left\{f_{x}\right\}_{x \in A}$ converges continuously to $h$ if the net $\left\{f_{x}\left(y_{\nu}\right)\right\}$ converges to $f(y)$ whenever the net $\left\{y_{\nu}\right\}_{\nu \in C}$ converges to $y$ (we omit the domain of composite nets). 
Consequently, $X^{I}$ is not normal ( $E$ is a closed subspace of $X^{I}$ which is not normal).

Theorem 2. Let $K$ be a countable $C W$-complex and $X$ a compact metrizable space. Then $K^{x}$ is a cosmic space (see footnote 4) whenever $K^{x}$ has the pointwise topology (or the compact-open topology ${ }^{8}$ ).

Proof. Let $M$ be the topological sum of the countable family $\left\{C_{n}\right\}_{n=1}^{\infty}$ of finite subcomplexes of $K$ (i.e. $\left.M=\cup_{n=1}^{\infty} C_{r} \times\{n\}\right)$. Then $M^{X}=\bigcup_{n=1}^{\infty} C_{n}^{\mathbb{X}}$, since $X$ is compact, and hence $M^{\boldsymbol{X}}$ with the pointwise topology, or the compact-open topology, is separable metrizable, because of Theorem 1 in [7].

Now we define a map $f: M \rightarrow K$ by $f(k, n)=k$ for each $(k, n) \in M$. Clearly $f$ is continuous (indeed $f$ is a quotient map). Then we define a map $\phi: M^{x} \rightarrow K^{x}$ by $\phi(h)=f \circ h$. Clearly $\phi$ is an on to map. Furthermore $\phi \mid C_{n}^{X}$ (the restriction of $\phi$ to $C_{n}^{X}$ ) is a homeomorphism and $M^{X}$ is the disjoint topological union of $\left\{C_{n}^{X}\right\}_{n=1}^{\infty}$. Hence $\phi$ is continuous whenever both $M^{x}$ and $K^{x}$ have the pointwise topology (or the compact-open topology).

THEOREм 3. There exists a 2-dimensional (in the covering sense) compact Hausdorff space $Y$ of weight ${ }^{9} 2^{N_{0}}$ such that $Y^{I}$ is not a normal space.

Proof. Let $X, F$ and $Z$ be the spaces constructed in the proof of Theorem 1, and let $Y$ be the Wallman compactification of $X$ (since $X$ is a normal space, $Y$ is also the Stone-Cěch compactification of $X$ see Exercise T, p. 169, in [6] for pertinent definitions and results). Then $Y$ has the same covering dimension of $X(\operatorname{dim} X=2)$ and weight" (since the topology of $X$ is carried onto a base for the topology of $Y$, the cardinality of the topology of $X$ is $2^{\aleph_{0}}$, and any base for the topology of $X$ must contain some $N(w, \sigma, \partial)$ for each real number $w$ ). Then we get that $X^{I}$ and hence $Z$ are subspaces of $Y^{I}$.

We show that $Z$ is an $F_{\sigma}$-subset of $Y^{I}$ : For each positive integer $n$, let $F_{n}=\left\{f_{w} \in F \mid-n \leqq w \leqq n\right\}$. Then $F_{n}$ is a closed subset of $Y^{I}$ we essentially repeat part (b) of the proof of Theorem 1 (keeping in mind that $Y^{I}$ is Hausdorff (so nets converge to at most one point) and $\{(x, y) \mid-n \leqq x \leqq n\}$ is a compact subspace of $Y$ for each $y \geqq 0)$. Then $Z$ is the union of countably many closed subsets of $Y^{I}$, since $Z-F$ is

${ }^{8}$ Actually, if $K^{x}$ has the compact-open topology then a stronger version of Theorem 2 is already known-see Definitions 1.1 and 1.2, and results (I) and (J) of $[8]$.

- The weight of a topological space $X$ is the minimum of the cardinal numbers of the open bases for the topology on $X$. 
countable, and hence $Z$ is an $F_{\sigma}$-subset of $Y^{I}$. Consequently $Y^{I}$ is not a normal space, since $F_{\sigma}$-subsets of normal spaces are normal and $Z$ (an $F_{\sigma}$-subset of $Y^{I}$ ) is not a normal space.

\section{BIBLIOGRAPHY}

1. R. Arens and J. Dugundji, Topologies for function spaces, Pacific J. Math. 1 (1951), 5-31.

2. C. J. R. Borges, On stratifiable spaces, Pacific J. Math 17 (1966), 1-16.

3. J. G. Ceder, Some generalizations of metric spaces, Pacific J. Math. 11 (1961), $105-125$.

4. J. Dugundji, An extension of Tietze's theorem, Pacific J. Math. 1 (1951), 353-367.

5. F. B. Jones, Concerning normal and completely normal spaces, Bull. Amer. Math. Soc. 43 (1937), 671-677.

6. J. L. Kelley, General topology, Van Nostrand, Princeton, N. J., 1955.

7. E. A. Michael, On a theorem of Rudin and Klee, Proc. Amer. Math. Soc. 12 (1961), 921.

8. - No-spaces, (to appear).

9. A. H. Stone, $A$ note on paracompactness and normality of mapping spaces, Proc. Amer. Math. Soc. 14 (1963) 81-83.

University of California, Davis 